\title{
Valoración de factores pronósticos de la supervivencia en una serie de 202 pacientes intervenidos por carcinoma de células renales
}

\author{
M. Ortiz Gorraiz, F.J. Vicente Prados, J.L. Rosales Leal, B. Honrubia Vílchez, \\ A. Martínez Morcillo, J.M. Cózar Olmo, E. Espejo Maldonado, M. Tallada Buñuel
}

Servicio de Urología. Hospital Universitario Virgen de las Nieves. Granada.

Actas Urol Esp 2005; 29 (2) 179-189

\section{RESUMEN}

VALORACIÓN DE FACTORES PRONÓSTICOS DE LA SUPERVIVENCIA EN UNA SERIE DE 202 PACIENTES INTERVENIDOS POR CARCINOMA DE CÉLULAS RENALES

Objetivos: Describir los principales factores pronósticos del carcinoma renal y estudiar su relación con la supervivencia especifica. Recoger datos demográficos, clínicos, diagnósticos y terapéuticos de los pacientes.

Material y métodos: Se revisaron 202 casos de carcinomas renales operados entre 1988-2003. Las variables valoradas respecto al paciente fueron: edad, sexo y presentación clínica. Se investigó la importancia del incidentaloma. Con respecto al tumor se valoró: estadio TNM, grado histológico, tamaño y afectación venosa. Con respecto al tratamiento: principales vías de abordaje y técnicas quirúrgicas y terapia adyuvante inmunoterápica.

Resultados: Un 60\% de los pacientes presentó enfermedad organoconfinada, un 10\% afectación nodal y un 7\% enfermedad metastásica. Un $42 \%$ se presentaron incidentalmente, con una significativa mejor supervivencia respecto a tumores sintomáticos. Un $42 \%$ de los pacientes $\mathrm{pN}+$ presentaron metástasis a distancia al diagnóstico y un tercio las desarrollaron durante el seguimiento. La supervivencia de los pacientes metastásicos sin tratamiento adyuvante fue inferior al año, mientras que un $20 \%$ de los tratados con inmunoterapia permanecían vivos a los 2 años.

Conclusiones: El carcinoma renal es un tumor con alta mortalidad, fundamentalmente en sus estadios más avanzados $\left(\mathrm{pN}^{+} \mathrm{o} \mathrm{pM}+\right)$. Pese a las nuevas modalidades de tratamiento, no se ha logrado mejorar sustancialmente la supervivencia.

Palabras Clave: Carcinoma de células renales. Factores pronósticos. Inmunoterapia. Supervivencia.

\section{ABSTRACT}

\section{SURVIVAL PROGNOSTIC FACTORS VALUATION ON A SERIES OF 202 PATIENTS WITH SURGICAL} TREATMENT OF RENAL CELL CARCINOMA.

Objectives: To describe renal cell carcinoma prognostic factors and set up the relationship with survival rates in this neoplasm. Likewise we show epidemiologic, clinical, diagnosis and therapeutic facts.

Material and method: We review 202 patients underwent surgical treatment for renal cell carcinoma and the following features were recorded: gender, age and presenting symptoms, especially incidentally discovered tumors; tumor-related factors like TNM tumor stage, tumor grade and venous involvement; therapy-related recorded were surgical techniques and cytokine-based therapy.

Results: $60 \%$ of the patients showed organ-confined disease, $10 \%$ of patients with renal cell carcinoma presented with nodal positive disease and $7 \%$ with systemic metastases. $42 \%$ of patients presenting incidental tumor, with survival rates substantially better than that for symptomatic patients. $42 \%$ of patients with nodal positive disease presented systemic metastases at diagnosis, and $30 \%$ at surveillance. Systemic metastases portend a particularly poor prognosis for patients with renal cell carcinoma, with 12-months survival rates that $0 \%$. Patients with cytokine-related therapy for metastatic disease presented 24 -months survival rates that $20 \%$.

Conclusions: Renal cell carcinoma remains a major source of mortality, basically at advanced disease (nodal positive disease or systemic disease), without a clear improvement of survival rates despite the newer therapy modalities. 
$\mathrm{E}^{\mathrm{i}}$ carcinoma de células renales (CCR) supone alrededor del 3\% de todos los tumores sólidos del adulto, con un incremento en su detección a nivel mundial del 1,5-5,9\% anualmente, habiendo aumentado su incidencia hasta en un $30 \%$ en la última década, relacionado todo ello con una mejora en las técnicas diagnósticas de imagen. Se trata de la tercera neoplasia más frecuente del tracto urinario, con 20.000 nuevos casos diagnosticados anualmente en la Unión Europea $^{1}$. Se conoce que la relación entre incidencia y mortalidad asociada a este tumor es la más alta de todos los cánceres urológicos ${ }^{2}$. En la actualidad no existen factores de riesgo claramente aceptados para el CCR, aunque varios estudios epidemiológicos indican que el tabaco, la obesidad y la exposición a ciertos metales pesados como el cadmio podrían favorecer el desarrollo de esta neoplasia ${ }^{2}$.

Afecta fundamentalmente a personas entre la $6^{\underline{a}}$ y $7^{\underline{a}}$ décadas de la vida, predominando en el varón. A pesar del aumento en los últimos años en la detección de tumores asintomáticos y organoconfinados con posibilidad de tratamiento más efectivo, las tasas de mortalidad por cáncer renal también se han incrementado ${ }^{3}$.

En la actualidad se han establecido algunos factores pronósticos del CCR relacionados con el tumor, el propio paciente o el tratamiento realizado, mientras que otros factores están siendo investigados y no existen datos suficientes en la literatura para su manejo práctico en la consulta diaria ${ }^{4}$.

Dentro de los factores pronósticos relacionados con el tumor, el estadio patológico fundamentalmente mediante la clasificación TNM es el factor que mejor predice la supervivencia libre de enfermedad de estos pacientes. En las últimas series publicadas, esta tasa de supervivencia fue del $90-100 \%$ para el estadio I, $75-95 \%$ para el estadio II, $60-70 \%$ para el estadio III y $15-30 \%$ para el estadio IV ${ }^{4}$.

Otro factor relacionado con la pieza quirúrgica es el grado nuclear, mediante la clasificación de Fuhrman o de la OMS. El tipo histológico del tumor también se ha correlacionado con la supervivencia, habiéndose establecido que patrones histológicos presentan mejor y peor pronóstico ${ }^{5}$. Así, dentro del CCR, el carcinoma de ductos colectores podría representar la variante con el pronóstico más desfavorable ${ }^{6}$. Dentro de los factores relacionados con el tumor, se están realizando grandes avances en cuanto a marcadores biomoleculares y citogenéticos, pero que en la actualidad no son utilizados de forma universal en la clínica para conocer la posible evolución de cada paciente en particular. Sí parece tener más interés para el Urólogo el reconocer aquellos factores relacionados con el paciente, y que pueden determinar el posible tratamiento $\mathrm{y}$ condicionar la ocasional terapia adyuvante. Si bien edad, género, raza y estatus socioeconómico no parecen poseer valor pronóstico ${ }^{4}$, sí han demostrado su influencia en la evolución de la enfermedad la forma de presentación clínica del tumor, el estado general del paciente y ciertas enfermedades genéticas predisponentes (por ejemplo. VHL) ${ }^{7}$. Actualmente se discute sobre el valor pronóstico de ciertos marcadores séricos como hemoglobina, PCR, $\beta-2$ microglobulina y fosfatasa alcalina entre otros ${ }^{8}$.

Por último, y en relación con los factores pronósticos relacionados con el tratamiento, la posibilidad de realizar la exéresis quirúrgica del tumor condiciona en gran modo la tasa de supervivencia de estos pacientes. Sin embargo, el gran problema sigue existiendo en los pacientes metastásicos, donde aún los mejores resultados que ha demostrado la inmunoterapia no alcanzan respuestas importantes a largo plazo ${ }^{1}$.

El tratamiento de elección del CCR localizado o localmente avanzado sigue siendo la cirugia ${ }^{9}$. Los casos de enfermedad metastásica, que en algunas series alcanzan un $25 \%$ de los pacientes al diagnóstico y hasta un $20-30 \%$ más tras el tratamiento quirúrgico durante su seguimiento, debido a su pobre pronóstico (supervivencia inferior al $10 \%$ a los 5 años), deben considerarse tributarios de inmunoterapia sistémica combinada adyuvante en el contexto de los actuales ensayos clínicos en Fase II y III, ya que la respuesta a la quimioterapia sistémica, incluso con los nuevos agentes, ha sido muy reducida $(2,5-5 \%)^{1}$.

\section{OBJ ETIVOS}

Los objetivos que se pretendían al realizar la revisión retrospectiva de nuestra serie de 202 CCR intervenidos en el Hospital Virgen de las 
Nieves de Granada fueron recoger los datos epidemiológicos básicos de la serie así como la distribución de los casos por estadios, intentando establecer que factores se asociaron a un peor pronóstico valorado como una reducción significativa de la supervivencia específica de enfermedad. Igualmente, del análisis de estos factores pretendemos extraer unas pautas de actuación en cuanto a seguimiento y necesidad de tratamiento adyuvante en función de como y cuando tienden a recurrir estos pacientes, con el fin de conseguir un diagnóstico precoz de la recidiva y poder optar a un tratamiento más temprano de ésta con una esperable mejor respuesta a la terapia.

\section{MATERIAL Y MÉTODOS}

Entre Enero de 1988 y Diciembre de 2003, 202 pacientes con carcinoma de células renales fueron sometidos a intervención quirúrgica en nuestro Servicio. Revisamos de forma retrospectiva esta serie de pacientes.

$\mathrm{El}$ rango de edad de los pacientes fue de 18 a 83 años, con una mediana de edad de 62 años. La relación hombre: mujer fue de aproximadamente 3:2 (129 y 73 respectivamente). El riñón derecho estuvo implicado en un $60 \%$ de los casos y el izquierdo en el restante $40 \%$. En 8 casos (4\%) el tumor se presentó de forma bilateral. Prácticamente en todos los casos se llegó al diagnóstico clínico mediante la ultrasonografía $(n=172)$ o la tomografía computerizada $(n=182)$, reservándose la utilización de las imágenes de resonancia magnética $(n=37)$ para los casos con sospecha de afectación venosa por trombo tumoral, y la arteriografía $(n=13)$ para planear la estrategia quirúrgica en algunos pacientes donde se propuso cirugía conservadora de parénquima renal. La nefrectomía radical fue la técnica quirúrgica más ampliamente realizada, con adrenalectomía $(33,6 \%)$ o sin ésta $(52,5 \%)$. La cirugía conservadora se realizó en casos seleccionados $(12,4 \%)$, principalmente mediante tumorectomía. La vía de abordaje fue mayoritariamente la subcostal anterior $(77,7 \%)$, dejando el acceso por lumbotomía para los casos de cirugía conservadora. En el 1,5\% de los casos se estableció la inoperabilidad del caso una vez realizada la laparotomía, aunque sí se tomaron muestras para estudio histopatológico. La media de seguimiento para esta serie de pacientes fue de 57 meses, con un rango de 3-185 meses. Desde el punto de vista del estado de los pacientes durante el seguimiento, un $5,9 \%(n=12)$ se perdieron para control, un $70,3 \%$ continúan vivos, un $16,3 \%$ fallecieron a causa del tumor renal y el restante $7,4 \%$ lo hicieron por otras causas. El análisis de los datos se realizó mediante el paquete informático SPSS versión 11.0.1 y las expectativas de supervivencia se calcularon con el método KaplanMeier. Una vez recogidos los datos y analizados de forma univariante aquellos factores que motivaron diferencias en el pronóstico de esta serie de pacientes, se realizó un análisis multivariante mediante Análisis de Regresión Múltiple de Cox.

\section{RESULTADOS}

En base al sistema de estadiaje de tumores TNM (UICC, 2.002), la Tabla 1 recoge los datos obtenidos de esta serie de pacientes en cuanto a su estadio pTpNM al diagnóstico. La supervivencia específica de enfermedad de esta serie fue del $90 \%$ a los 12 meses, $84 \%$ a los 5 años y $81 \%$ a los 10 años. El tiempo medio de supervivencia en los pacientes fallecidos por la neoplasia renal $(16,3 \%)$ fue de 24 meses (rango 2-140).

La supervivencia total en el momento del estudio fue del $70^{`} 3 \%$.

La mortalidad perioperatoria fue del $1 \%(n=2)$.

De forma global la Tabla 2 recoge la distribución de los pacientes por estadio tumoral y la supervivencia específica de enfermedad a los 5 años.

Tabla 1

Descripción de la implicación nodal y metástasis a distancia según estadio pT

\begin{tabular}{|c|c|c|c|c|c|c|}
\hline \multicolumn{3}{|c|}{ Estadio } & \multicolumn{2}{|l|}{ pN+ } & \multicolumn{2}{|l|}{$\mathbf{M}+$} \\
\hline & $\%$ & $\bar{n}$ & $\%$ & $\bar{n}$ & $\%$ & $\mathbf{n}$ \\
\hline pTla & 13,4 & 27 & - & - & - & - \\
\hline pT1b & 25,7 & 52 & - & - & - & - \\
\hline pT2 & 22,3 & 45 & 9,5 & 2 & - & - \\
\hline pT3a & 20,8 & 42 & 19 & 4 & 20 & 3 \\
\hline pT3b & 12,9 & 26 & 28,5 & 6 & 46,6 & 7 \\
\hline pT3c & 0,5 & 1 & - & - & - & - \\
\hline pT4 & 4,5 & 9 & $42-8$ & 9 & 33,3 & 5 \\
\hline Total & 100 & 202 & $10^{-4}$ & 21 & 744 & 15 \\
\hline
\end{tabular}


Tabla 2

Estadio tumoral y supervivencia a 5 años

\begin{tabular}{lclcc}
\hline Hallazgo & $\begin{array}{c}\text { Estadio } \\
\text { Robson }\end{array}$ & TNM (2002) & \% & $\begin{array}{c}\text { Supervivencia } \\
\mathbf{5} \text { años (\%) }\end{array}$ \\
\hline Órgano confinado & I & pT1-2NOMO & 60,4 & 98 \\
Grasa perirrenal o adrenal & II & pT3ANOMO & 17,8 & 75 \\
Afectación venosa & IIIA & pT3b-cNOMO & 9,4 & 75 \\
Localmente avanzado & IVA & pT4NOMO & - & - \\
Afectación linfática & IIIB & pTxN+MO & 5,5 & 50 \\
Metástasis sistémicas & IVB & pTxNxM+ & 6,9 & 10 \\
\hline
\end{tabular}

En el grupo de pacientes con incidentaloma, el desarrollo de metástasis durante su seguimiento se presentó en el 4,8\% de los casos, siendo esta cifra del $10,1 \%$ en los pacientes sintomáticos al diagnóstico del CCR. Desde el punto de vista de la supervivencia a corto y largo plazo encontramos diferencias igualmente entre estos dos grupos de pacientes (Logrank<0,001), de forma que a

A continuación valoramos algunos de los factores pronósticos que pensamos han tenido un mayor impacto en la supervivencia de esta serie de pacientes, y recogemos algunos resultados más en profundidad.

\section{Presentación clínica}

Desde el punto de vista de la presentación clínica, el 24\% de los pacientes consultó por dolor, el $24 \%$ por hematuria, el $8 \%$ de los pacientes se diagnosticaron en el contexto de un síndrome constitucional, el 1\% presentó shock hipovolémico (S. Wünderlich) y el $1 \%$ como síntomas/ signos derivados de metástasis óseas o pulmonares.

En el $42 \%$ de los casos fue un hallazgo incidental en el transcurso de un estudio ecográfico o tomográfico fundamentalmente. Con una edad media de 60 años, este grupo estuvo compuesto por 42 hombres y 42 mujeres. La distribución de esta subpoblación de pacientes en base al estadiaje TNM se ilustra en la Tabla 3.

Tabla 3

Descripción de la implicación nodal y metástasis a distancia según estadio pT en pacientes con incidentaloma renal

\begin{tabular}{|c|c|c|c|c|c|c|}
\hline \multicolumn{3}{|c|}{ Estadio } & \multicolumn{2}{|l|}{ pN+ } & \multicolumn{2}{|c|}{ M+ } \\
\hline & $\%$ & $\bar{n}$ & $\%$ & $n$ & $\%$ & $\bar{n}$ \\
\hline pTla & 22,6 & 19 & - & - & - & - \\
\hline pT1b & 33,3 & 28 & - & - & - & - \\
\hline pT2 & 20,2 & 17 & 33,3 & 1 & - & - \\
\hline pT3a & 17,9 & 15 & 66,6 & 2 & - & - \\
\hline pT3b & 6 & 5 & & - & - & - \\
\hline pT3c & - & - & - & - & - & - \\
\hline pT4 & - & - & - & - & - & - \\
\hline Total & 100 & 84 & 3,5 & 3 & 0 & 0 \\
\hline
\end{tabular}

los 12,60 y 120 meses para los incidentalomas fue del 98\%, 97\% y 95\% siendo para los pacientes sintomáticos del 87\%, 75\% y 70\% respectivamente. Se investigó igualmente cual había sido la evolución de los CCR incidentales a lo largo de los años, distribuyendo a esta serie de 202 pacientes en tres grupos en función de la fecha de la intervención: Grupo 1 ( $n=39 ; 1988-1993)$, Grupo 2 (n=79;19941998) y Grupo 3 ( $n=84 ; 1999-2003)$. Dichos grupos fueron comparables en cuanto a edad y sexo de los pacientes. Se determinó para cada grupo que porcentaje de pacientes se presentaron de forma incidental, así como la distribución de diferentes factores de mal pronóstico (grado nuclear alto, pT3a-pT4, pN+ y M+), lo cual queda recogido en la Tabla 4. Calculamos mediante análisis de Kaplan-Meier la supervivencia específica de enfermedad para cada uno de los grupos, obteniéndose a los 5 y 10 años las siguientes cifras: Grupo 1 (83\% y 78\%); Grupo 2 (83\% y 75\%); y Grupo 3 (85\% y 85\%).

En nuestra serie la evolución del CCR incidental ha sido creciente en los últimos 15 años, con un ascenso desde el 30`8\% en el primer quinquenio, hasta el 45,2\% en los últimos cinco años. Sin embargo esto no se ha traducido en una reducción significativa de los tumores con factores de mal pronóstico ni una mejoría evidente de la supervivencia global de la serie.

\section{Estadio $p T$}

En la Tabla 1 se recoge la distribución de esta serie de pacientes en función de su categoría pT, así como el número de pacientes en los que se estableció positividad para adenopatías o metástasis a distancia al diagnóstico. 
Tabla 4

Evolución temporal del incidentaloma recogiendo diferentes factores pronósticos

\begin{tabular}{|c|c|c|c|c|c|c|c|c|c|c|c|}
\hline & \multirow[b]{2}{*}{$\mathbf{n}$} & \multicolumn{2}{|c|}{ Incidental } & \multicolumn{2}{|c|}{ G3-4 } & \multicolumn{2}{|c|}{ рT3a-pT4 } & \multicolumn{2}{|c|}{ pN+ } & \multicolumn{2}{|c|}{ M+ } \\
\hline & & $\bar{n}$ & $\%$ & $\bar{n}$ & $\%$ & $n$ & $\%$ & $\mathbf{n}$ & $\%$ & $n$ & $\%$ \\
\hline $\begin{array}{c}\text { GRUPO 1 } \\
(1988-1993)\end{array}$ & 39 & 12 & $30{ }^{-} 8$ & 14 & $35^{\circ} 9$ & 15 & $38^{-4}$ & 2 & 5,1 & 3 & 7,7 \\
\hline $\begin{array}{c}\text { GRUPO } 2 \\
(1994-1998)\end{array}$ & 79 & 34 & 43 & 22 & $28^{\circ} 6$ & 36 & $45^{\circ} 6$ & 9 & 11,4 & 4 & 5,1 \\
\hline $\begin{array}{c}\text { GRUPO } 3 \\
(1999-2003)\end{array}$ & 84 & 38 & 45,2 & 31 & 369 & 27 & 32,2 & 11 & $11^{`} 9$ & 8 & $9 ־ 5$ \\
\hline Total & 202 & 84 & 41,6 & 67 & 33,2 & 78 & 38,6 & 21 & 10,3 & 15 & 7,4 \\
\hline
\end{tabular}

Como sabemos, la existencia de ganglios positivos o metástasis en otros órganos determina la supervivencia. Por este motivo quisimos establecer cual era la evolución de aquellos pacientes sin adenopatías positivas y no metastásicos al diagnóstico, en función de su estadio pT (Fig. 1). Como vemos no existieron diferencias entre aquellos pacientes con tumor organoconfinado (pT1a/b-pT2) con una supervivencia a los 5 años del $95-100 \%$, descendiendo esta al 75\% cuando se infiltraba la grasa o se afectaba la glándula adrenal o de un $81 \%$ cuando existía afectación venosa por trombo tumoral.

\section{Estadio $p N$}

Como se observa en la Tabla 1, el 10,4\% de los pacientes fue $\mathrm{pN}^{+}$, detallándose en ésta como se

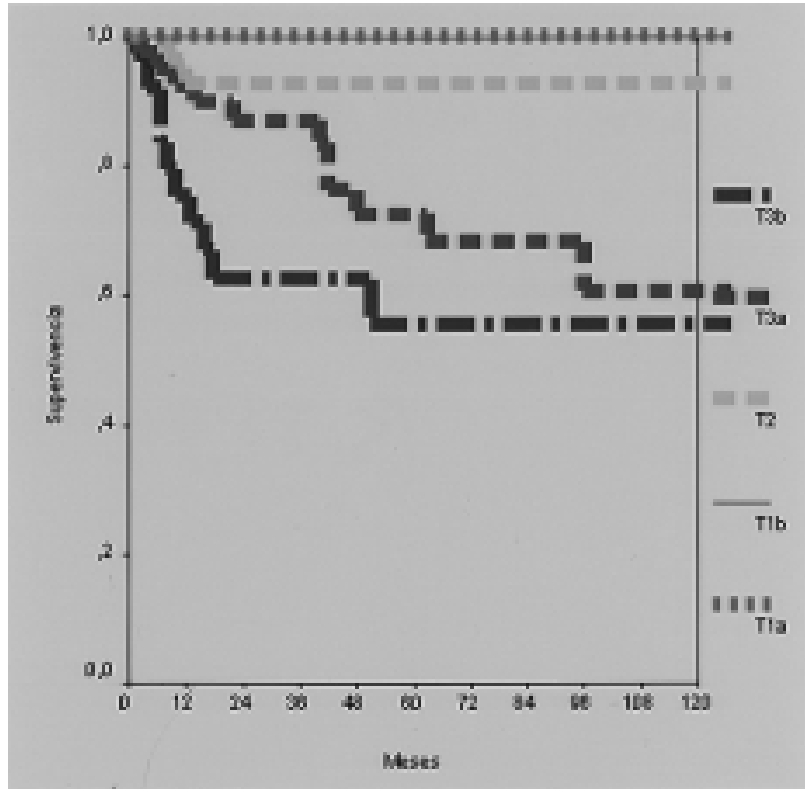

FIGURA 1. Supervivencia por estadio pT en pacientes pNOMO. distribuyeron estos pacientes por estadio pT. De los 21 pacientes con adenopatías positivas, el $42 \%$ presentaban sincrónicamente metástasis a distancia. Del resto de pacientes, hasta un 33\% desarrollaron metástasis en órganos distantes durante su seguimiento.

La supervivencia de los pacientes $\mathrm{pN}+$ a los 6 , 12 y 24 meses fue del 70\%, 40\% y 32\% respectivamente, muy inferior a la de aquellos pacientes que no presentaron adenopatias positivas (Fig. 2).

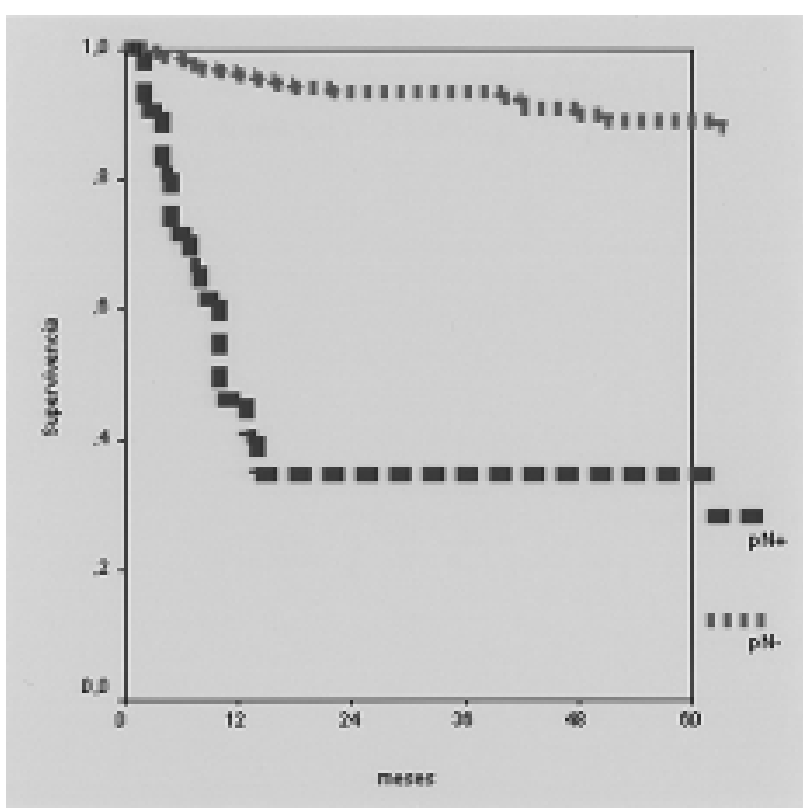

FIGURA 2. Supervivencia según la presencia o no de ade nopatias positivas.

\section{Estadio $M$}

En nuestra serie, al diagnóstico, el 7,4\% de pacientes fue metastásico, siendo un tercio de estos casos en múltiples localizaciones (al menos en 2 órganos a distancia). Por frecuencia, las 
metástasis fueron: pulmonares (40\%), óseas (33\%), hepáticas (20\%), SNC (13\%), retroperitoneo $(13 \%)$, peneanas $(6 \%)$, peritoneales $(6 \%)$ y adrenales (6\%). Durante el seguimiento del subgrupo de pacientes no metastásicos al diagnóstico $(n=187)$, el 8,5\% $(n=16)$ de éstos desarrolló enfermedad a distancia. En 4 casos se trató de pacientes $\mathrm{pN}+$ como ya se indicó previamente. Entre los pacientes pNO que desarrollaron metástasis, encontramos la siguiente distribución por estadios pT: pT1b 16\%, pT2 25\%, pT3a 43\% y pT3b-c 16\%. Las principales localizaciones de metástasis en el seguimiento fueron hueso (36\%), pulmón (27\%), hígado (27\%), SNC (5\%) y cutánea (5\%). El tiempo medio hasta la detección de la/s metástasis fue de 29 meses (rango 4-129 meses). La supervivencia media tras el diagnóstico de enfermedad metastásica fue de 4 meses (rango 110 meses).

De los 15 pacientes metastásicos al diagnóstico, 9 se sometieron a inmunoterapia. Al valorar la supervivencia de los pacientes con enfermedad a distancia, encontramos diferencias en cuanto a si fueron o no sometidos a inmunoterapia, de forma que la supervivencia máxima de los pacientes metastásicos sin tratamiento adyuvante fue de 7 meses, mientras que en los pacientes sometidos a inmunoterapia la supervivencia a los 6,12 y 24 meses fue del 65\%, 35\% y $20 \%$ respectivamente.

\section{Afectación Venosa}

Un total de 30 pacientes $(14,8 \%)$ presentaron afectación venosa por trombo tumoral (v. renal $9,4 \%$; v. cava $5,4 \%$ ).

En el $73 \%$ de los casos el riñón afectado fue el derecho. El 30\% de los pacientes con afectación vascular por trombo tumoral presentaron adenopatías positivas, y un $26 \%$ fueron metastásicos al diagnóstico. De los 22 pacientes no metastásicos al diagnóstico, un 18\% desarrollaron metástasis en su evolución.

En cuanto a la supervivencia especifica de enfermedad (Fig. 3), los pacientes con trombo tumoral presentaron peores resultados que aquellos sin afectación venosa, siendo ésta del $55 \%$ y $42 \%$ frente al $89 \%$ y $85 \%$ a los 5 y 10 años respectivamente (Log-rank $<0,00)$.

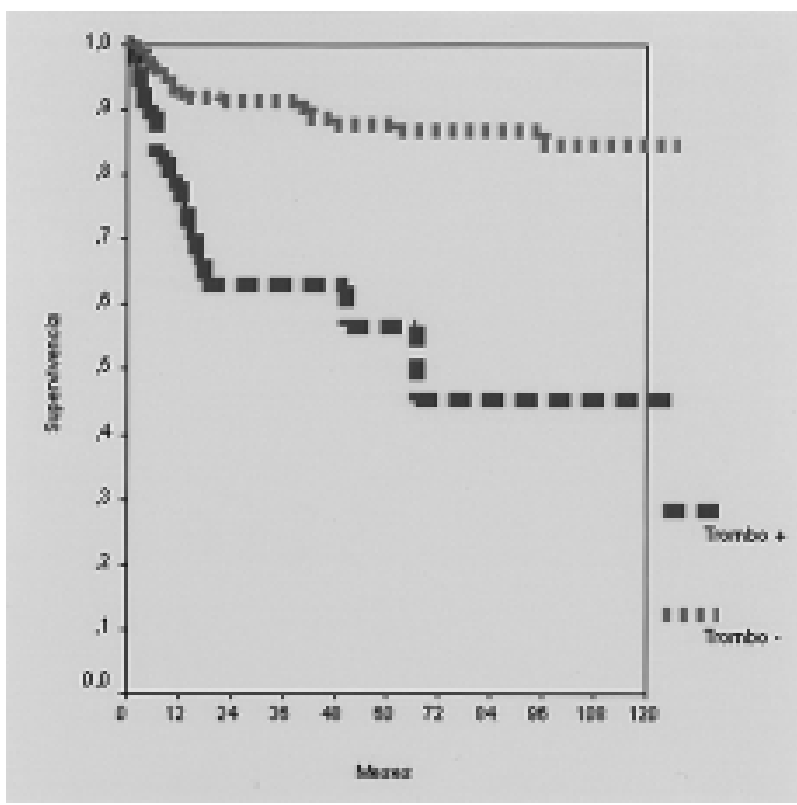

FIGURA 3. Supervivencia según afectación o no de trombosis venosa tumoral.

\section{Grado histológico}

Al valorar el grado histológico (Furhman), los pacientes se distribuyeron del siguiente modo: G1-2 67\% y G3-4 33\%. En el primer grupo de pacientes sólo el 1,5\% presentó metástasis o adenopatías positivas al diagnóstico, mientras que esta cifra ascendió hasta un 20\% y $25 \%$ respectivamente en los tumores de alto grado (G3-4).

Al valorar la supervivencia de ambos grupos (Fig. 4) también se encontraron diferencias significativas (Log-rank<0,0001), con supervivencias a los 12, 60 y 120 meses del 96\%, 94\% y $92 \%$ respectivamente para los pacientes con tumores de bajo grado (G1-2), bajando estas cifras hasta el $82 \%, 64 \%$ y $52 \%$ para los de alto grado (G3-4).

\section{Recidiva local tras Nefrectomía Radical.}

Del total de pacientes sometidos a nefrectomía radical, un 2,3\% $(n=4)$ sufrieron recidiva en el lecho quirúrgico. Excepto en un caso (pT2), el resto de pacientes habían presentado afectación de la grasa perirrenal en la pieza (pT3a). Un paciente fue manejado con cirugía de rescate mediante exéresis de la masa, con buen resultado hasta la fecha. En el resto de casos se realizó inmunoterapia de rescate con resultados de supervivencia dispares (4-15 meses). 


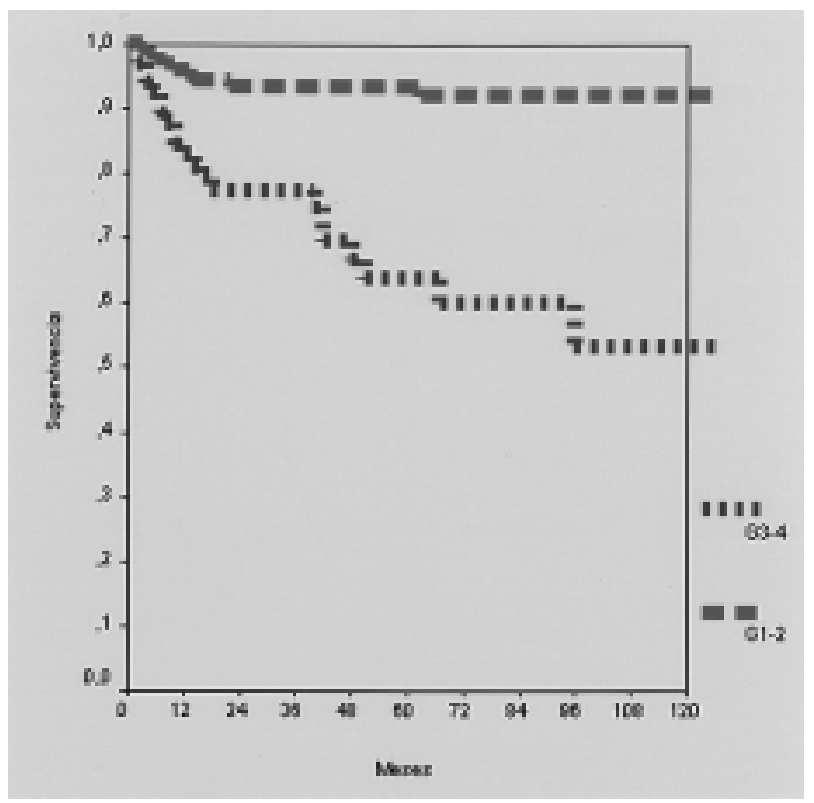

FIGURA 4. Supervivencia según grado histológico tumoral.

\section{Cirugía conservadora}

Como se indicó anteriormente, 25 pacientes se sometieron a cirugía conservadora $(12,4 \%)$, en 22 casos mediante tumorectomía y en otros 3 mediante nefrectomía parcial. No existieron diferencias con respecto a edad de los pacientes ni a días de ingreso con respecto a la serie general. Desde el punto de vista clínico, el 68\% de los casos fue un incidentaloma. En cuanto a la pieza quirúrgica, el tamaño tumoral medio fue de 377 $\mathrm{cm}$, con un grado tumoral G1-2 en 24 de los 25 pacientes intervenidos. Sólo 3 pacientes se categorizaron como pT2, siendo el resto pT1. En la actualidad, y con un seguimiento medio de 60 meses, el $92 \%$ de los pacientes sigue vivo, con un paciente perdido para seguimiento y otro fallecido por otra causa no oncológica. Hemos encontrado un caso de recidiva local tras la cirugía conservadora, con buen resultado posterior tras la nefrectomía radical.

\section{CCR en pacientes adultos jóvenes.}

Un total de 30 casos (14,9\%) tenían entre 18 y 45 años de edad (media=39 años). Cuando comparamos los pacientes mayores de 45 años con aquellos de edad igual o menor a 45 años, no encontramos diferencias significativas en cuanto a la forma de presentación (incidental $=40 \%$ Vs $46 \%, \mathrm{p}=0,37)$, tamaño tumoral $(7,7 \mathrm{Vs} 6,7 \mathrm{~cm}$, $\mathrm{p}=0,23$ ), grado tumoral (G1-2=64\% Vs $80 \%$, G3$4=35 \%$ Vs $20 \%$, p=0,089) ni finalmente en cuanto al estadio TNM (1997): pT1-2=58\% Vs 76\%, $\mathrm{p}=0,062 ; \mathrm{pT} 3=29 \%$ Vs $13 \%, \mathrm{p}=0,064 ; \mathrm{pN}+=5 \% \mathrm{Vs}$ $3 \%, \mathrm{p}=0,58 ; \mathrm{M}+=5 \%$ Vs $6 \%, \mathrm{p}=0,85$. Para un seguimiento medio de 58 y 52 meses respectivamente, no aparecieron diferencias significativas en cuanto al desarrollo de metástasis a distancia $(8 \%$ vs $3 \%, p=0,3)$ ni en cuanto a la supervivencia libre de enfermedad (83\% Vs $86 \%$; Log rank test $\mathrm{p}=0,7$ ).

Otros datos de esta serie.

Desde el punto de vista del tumor, el tamaño medio de este fue de 7,5 cm (rango 1-20 cm). Por su localización principal encontramos un $36 \%$ en polo superior, un $35 \%$ mesorrenales y un $29 \%$ polares inferiores.

La estancia media de los pacientes tras la cirugía fue de 8 días.

Tras la intervención, un $81 \%$ de los pacientes conservaron adecuada función renal, mientras que un $14 \%$ desarrollaron insuficiencia renal crónica en diversos grados, requiriendo su entrada en programa de hemodiálisis un $5 \%$ de los pacientes.

\section{Análisis multivariante.}

Los datos del análisis multivariante mediante Regresión Múltiple de Coxse presentan en la Tabla 5. Destacamos, de este estudio multivariante, que únicamente la presencia de metástasis a distancia y el estadio pT se mantuvieron como factores independientes de supervivencia en esta serie.

\section{DISCUSIÓN}

Presentamos una serie de 202 casos de CCR intervenidos que representa una población creemos aceptable para su estudio y análisis estadístico. La composición demográfica en cuanto a distribución por edad y sexo no varía con respecto a otras series publicadas ${ }^{10,11}$. Si se presentó una mayor incidencia de tumores en adultos jóvenes con respecto a otras series publicadas, si bien esto no supuso una ventaja significativa con respecto a la supervivencia de este grupo de pacientes como si se ha detectado en estas series ${ }^{12}$. 
Tabla 5

Análisis multivariante de los datos

\begin{tabular}{lcccc}
\hline & \multirow{2}{*}{ Sig. } & \multirow{2}{*}{ Exp(B) } & \multicolumn{2}{c}{ 95\% IC para exp (B) } \\
\cline { 3 - 5 } & & & Inferior & Superior \\
\hline Grado &, 506 & 1,384 &, 534 & 3,603 \\
pT &, 0006 & 8,261 & 2,535 & 26,920 \\
pN &, 517 & 1,453 &, 469 & 4,502 \\
M &, 00015 & 8,472 & 2,806 & 25,579 \\
Trombo &, 859 &, 927 &, 399 & 2,153 \\
\hline
\end{tabular}

Destaca la mayor afectación del riñón derecho sobre el contralateral (3:2), superior a otras series más amplias publicadas ${ }^{13}$, aunque no ha sido descrito como un factor pronóstico por si mismo 4 .

En cuanto a las pruebas diagnósticas en el carcinoma de células renales, pensamos que la técnica de elección en el diagnóstico, estadiaje locorregional y en la detección de metástasis a distancia es la Tomografía Computerizada, que incluso permite sospechar en manos expertas la implicación venosa tumoral que podría ser confirmada mediante la $\mathrm{RMN}^{14}$. No utilizamos de rutina la gammagrafía ósea, sólo en casos sospechosos de metástasis óseas (dolor o fostatasa alcalina elevada) tanto al diagnóstico como durante el seguimiento del paciente. En casos seleccionados puede ser de utilidad la realización de arteriografía con anterioridad a la cirugía conservadora, si bien no siempre será imprescindible. Igualmente, el estudio isotópico renal, como arsenal diagnóstico, permite en pacientes afectos de insuficiencia renal crónica previa a la intervención, el decidir el planteamiento quirúrgico en base a la funcionalidad de ambas unidades renales.

Reconocemos que si bien el tipo histológico de CCR supone un criterio pronóstico, no hemos incluido este factor al no disponer de él en todos los casos. Si pensamos que es un criterio importante a la hora de valorar pacientes de alto y bajo riesgo para la recidiva tumoral, así como prever la posible respuesta biológica del tumor a ciertas terapias adyuvantes como la inmunoterapia. En esta serie no se incluyeron tumores renales diferentes del CCR.

En referencia al procedimiento quirúrgico en pacientes afectos de CCR, defendemos la realización de nefrectomía radical por vía subcostal anterior, que permite un buen control vascular, cuestión fundamental en casos de masas grandes con o sin afectación venosa por trombo tumoral. Igualmente creemos que es importante la exéresis por rutina del tejido adenopático que rodea a los grandes vasos retroperitoneales, al menos con una actitud diagnóstica, ya que como hemos visto en el apartado anterior, la migración de un paciente desde un estadio inferior hasta otro con implicación nodal supone un empobrecimiento muy importante de su pronóstico. En cuanto al beneficio terapéutico de la linfadenectomía permanece aun sin esclarecer, defendiendo en la actualidad algunos autores la linfadenectomía extensa como un medio para aumentar las expectativas de supervivencia de aquellos enfermos con adenopatías positivas ${ }^{15}$. Creemos que a falta de la publicación de datos concluyentes con respecto a algún estudio prospectivo que compare la linfadenectomía extensa versus su no realización en cuanto a la valoración de morbimortalidad asociadas y supervivencia de los diferentes grupos de pacientes, la exéresis del tejido linfático regional permite una mejor estadificación del paciente y puede, al menos, ayudar en la toma posterior de actitudes de seguimiento y de posibles terapias adyuvantes.

De forma similar a otras series publicadas, en la nuestra se puede apreciar un ascenso progresivo en el número de pacientes sometidos a cirugía por CCR, lo cual esta en concordancia con otros datos demográficos sobre el aumento de la incidencia en este tumor ${ }^{2}$.

Esta revisión de casos no pretendió investigar posibles factores epidemiológicos de riesgo para el CCR (tabaco, obesidad, hipertensión arterial, alimentación), cuestión hoy día que continúa provocando debates entre diferentes grupos de autores ${ }^{10}$. Si creemos importante determinar que agentes pueden estar relacionados con la aparición del CCR con el fin de poder realizar prevención primaria en este tipo de tumores.

Al revisar nuestros datos y compararlos con otros publicados ${ }^{10}$ en relación con la presentación clínica del tumor, observamos como cada vez es menos frecuente la clásica triada de dolor en flanco, hematuria y masa abdominal palpable como resultado de la disminución en el porcentaje de casos con diagnóstico tardío de la enfermedad. 
De forma general nuestra serie muestra un menor número de casos de dolor en flanco $(25 \%)$ y hematuria (24\%) que los reflejados en la literatura $(40 \% \text { y } 40-60 \% \text {, respectivamente })^{2}$. En cuanto a las diferencias de supervivencia especifica de enfermedad entre los pacientes sintomáticos a la presentación frente a aquellos con masas detectadas incidentalmente, encontramos unas cifras a los 5 años del $75 \%$ y $97 \%$ respectivamente, con una clara ventaja en los pacientes asintomáticos al diagnóstico. Datos similares en cuanto a la diferencia en las tasas de supervivencia las obtiene Pantuck y cols en una reciente serie publicada ${ }^{16}$, así como en series españolas ${ }^{17}$.

Nos parece también relevante destacar como en la evolución histórica de esta serie el tumor incidental ha ido incrementándose en los últimos 15 años (del 30\% al 45\%), y como este aumento en los incidentalomas no se ha traducido en una reducción evidente del porcentaje de pacientes con factores de mal pronóstico asociados al CCR, fundamentalmente enfermedad localmente avanzada, $\mathrm{pN}+\mathrm{o} \mathrm{M}+$ (ver Tabla 3), lo cual aunque paradójico, está en consonancia con otras series revisadas en las cuales el aumento en el diagnóstico incidental de tumores presintomáticos se acompañó de un incremento en las tasas de mortalidad por cáncer de riñón ${ }^{3}$.

Otros factores clínicos no mesurados en esta serie pero que creemos de importancia en el pronóstico de esta enfermedad son la perdida de peso mayor del $10 \% \mathrm{y}$ un pobre performance status al diagnóstico, que algunos autores han demostrado que poseen un impacto negativo en la supervivencia de los pacientes ${ }^{18}$, aparte de reducir la tolerabilidad del paciente a terapias agresivas ya sean quirúrgicas o no.

Los factores pronósticos relacionados con el tumor se consideran los más importantes para predecir la posible evolución de los pacientes con CCR. En nuestro grupo pensamos que los criterios TNM para el estadiaje del tumor renal son los más adecuados para categorizar a estos pacientes. En esta serie destaca un mayor porcentaje de pacientes con enfermedad organoconfinada $(60 \%)$ que en otras series ${ }^{11}$, lo cual podría explicar los mejores resultados de supervivencia especifica de enfermedad a largo plazo con respecto a datos de la literatura. Por el contrario, presentamos una serie con un porcentaje de pacientes metastásicos al diagnóstico $(7,4 \%)$ inferior a otras series donde se describen cifras de hasta el $20-25 \%{ }^{4}$ ). De igual forma ocurre con los pacientes que presentaron afectación nodal metastásica, si bien esto lo puede explicar el hecho que en esta serie todos los pacientes catalogados como $\mathrm{N}+$ eran en realidad $\mathrm{pN}^{+}$, mientras que en otras series también se incluyeron pacientes con adenopatías significativas en estudios radiológicos.

Un dato destacable de esta revisión es cómo en pacientes con $\mathrm{pN}+$, tanto al diagnóstico o bien durante el seguimiento, presentaron metástasis a distancia $(42,8 \%$ y $33,3 \%$ respectivamente), de forma que al valorar la serie obtuvimos que prácticamente las $3 / 4$ partes de los pacientes $\mathrm{pN}^{+}$ presentaron en uno $\mathrm{u}$ otro momento metástasis en otros órganos. Esto, junto con la importante reducción en la supervivencia de los pacientes con enfermedad nodal con respecto a los que no la presentan $(32 \%$ vs. $95 \%$ respectivamente a los 2 años) obliga a un manejo de este grupo de pacientes de forma muy similar a los pacientes metastáticos, en cuanto a la estrategia de controles posquirúrgicos así como al valorar a los pacientes como candidatos a posible tratamiento adyuvante.

De nuestros datos también se puede establecer que si bien el riesgo de desarrollar metástasis a distancia en pacientes sin afectación nodal al diagnóstico se incrementa con el estadio pT, hasta un $31 \%$ de éstas, aparecieron en pacientes con tumor organoconfinado (pT1-2), lo cual ha de ser tenido en cuenta a la hora de proponer un protocolo de seguimiento para este tipo de tumores.

Otro punto que permanece aun sin aclarar es el valor pronóstico real de la implicación venosa por trombo tumoral. En nuestros pacientes, aunque la presencia de trombo tumoral supuso una clara disminución de la supervivencia, al realizar el análisis estadístico multivariante, este factor perdió su significación. Es posible, tal y como proponen algunos autores, que la sola presencia de trombo tumoral en la luz vascular que generalmente será resecable no sea el condicionador del peor pronóstico de algunos de estos pacientes, sino que esta peor supervivencia este 
en relación con la microinvasión del endotelio vascular $^{19}$. Volviendo al análisis multivariante de nuestros datos, y en relación con la afectación nodal, pensamos que en este caso la perdida de significación estadística de $\mathrm{pN}+$ como factor pronóstico puede estar en relación al reducido número de pacientes que componen este grupo, así como a la dispersión que de estos se hace al distribuirlos en los diferentes estadios patológicos.

En vistas a futuros estudios, y centrándonos concretamente en los avances acerca de los diferentes marcadores moleculares en el $\mathrm{CCR}$ en relación con la utilización de distintos tipos de inmunoterapias, todas ellas encaminadas a estimular/aumentar la presentación antigénica a los linfocitos T, nuestro grupo está realizando diferentes análisis del infiltrado inflamatorio celular tanto del tumor, interfase tumoral y sangre periférica, con el fin de poder establecer diferencias entre distintos grupos de pacientes e intentar relacionarlas con la posible respuesta de estos pacientes a la inmunoterapia (datos no publicados). Creemos que estas investigaciones son necesarias ya que a pesar de los avances de los últimos años, las expectativas que se habían abierto en un principio en relación a este tipo de terapias se están viendo parcialmente reducidas. Esto es así porque, si bien en algunos pacientes la aplicación del protocolo inmunoterápico ha conseguido la regresión completa del tumor, en la mayoría ha fracasado. Entre los factores de este fracaso, se atribuye, fundamentalmente, el que habitualmente se suministran estos tipos de inmunoterapias en los últimos estadios de la enfermedad tumoral. Nosotros pensamos, que quizás, entre estos factores, también se encuentre una inadecuada selección de los pacientes, dado que en algunos de ellos esta terapia no puede ni se debe realizar, al no conocerse previamente cual es la situación inmunológica del paciente ni la presencia de determinados marcadores en el tumor, como serían la presencia de moléculas HLA de clase $\mathrm{I}^{20}$.

\section{CONCLUSIONES}

El carcinoma de células renales es en la actualidad un tumor con una incidencia en aumento, asociado esto a una mayor mortalidad por esta enfermedad a pesar de su cada vez más temprano diagnostico en fases localizadas y presintomáticas de la enfermedad. A pesar de los múltiples factores pronósticos que se han intentado asociar a este tumor, creemos que tanto la presencia de metástasis a distancia como la afectación nodal son los principales condicionantes de mal pronostico, sin que en la actualidad se pueda establecer qué peso relativo poseen cada uno de estos factores por separado, sino que mas bien habría que pensar en ellos como factores aditivos a la hora de valorar las expectativas de supervivencia del paciente.

Es necesario seguir investigando qué grupo de pacientes puede ser el mejor candidato a beneficiarse de tratamientos adyuvantes a la cirugía así como poder prever cuales de éstos responderán mejor a estas terapias actualmente en estudio, y para conseguir este fin será necesario establecer unas claras relaciones entre aquellos factores clínicos que actualmente venimos utilizando y los marcadores moleculares que suponen finalmente la vía de actuación de muchos de los tipos de inmunoterapia que hoy en día se emplean.

\section{REFERENCIAS}

1. Kirkali $Z$, Tuzel E, Mungan Mu. Recent advances in kidney cancer and metastatic disease. BJU International 2001; 88:188-824.

2. Kirkali Z, Öbek C. Clinical aspects of renal cell carcinoma. EAU Update Series 2003;1:189-196.

3. Chow Wh, Devesa Ss, Fraumeni Jr Jf. Rising incidence of renal cell cancer in the United States. JAMA 1999; 281: 1628-1631.

4. Lang H, Jacqmin D. Prognostic factors in renal cell cancer. EAU Update Saries 2003; 1: 215-219.

5. Störkel S, Eble JN, Adlakha K, Amin M, Blute ML, Bostwick DG, Darson M, Delahunt B, Iczkowski K. Classification of renal cell carcinoma. Cancer 1997;80: 987-989.

6. Ortiz Gorraiz M, Rosales Leal JL, Tallada Buñuel M, et al. Collecting duct carcinoma of the kidney with retroperitoneal lymph mass. Arch Esp Urol 2004;57:179-182.

7. Tsui KH, Shvarts O, Smith RB, Figlin R, de Kernion JB, Belldegrun A. Renal cell carcinoma: prognostic significance of incidentally detected tumors. J Urol 2002;163: 426-430.

8. Rubio Briones J, Iborra Juan I, Climent MA, Lopez Guerrero JA, Solsona Narbon E, et al. Utilidad de marcadores séricos del carcinoma renal. Actas Urol Esp 2004; 28:381-386

9. Van Poppel H, Deroo F, Joniau S. Open surgical treatment of localised renal cell cancer. EAU Update Series 2003;1: 220-225. 
10. Novick AC, Campbell SC; Renal tumors. En: Walsh PC, Retik AB, Vaughan ED, Wein AJ eds: Campbell's Urology, 8th ed, Pp 2672-2731. Saunders Ed., Philadelphia, 2002.

11. Giberti C, Oneto F, Martorana G, et al. Radical nephrectomy for renal cell carcinoma: long-term results and prognostic factors on a series of 328 cases. Eur Urol 1997;31: 40-48.

12. Argüelles Salido E, Medina López RA, Congregado Ruiz CB, Cayuela Domínguez A. Pascual de Pobil Moreno JL. Análisis de las neoplasias renales en adultos menores de 40 años. Actas Urol Esp 2004;28:335-340.

13. Kontak JA, Campbell SC. Prognostic factors in renal cell carcinoma. Urol Clin N Am 2003;30:467-468.

14. Israel GM, Bosniak MA. Renal imaging for diagnosis and staging of renal cell carcinoma. Urol Clin $\mathrm{N}$ Am 2003;30:499-514.

15. Guilliani L, Giberti C, Martorana G, et al. Radical extensive surgery for renal cell carcinoma: long-term results and prognostic factors. J Urol 1990;143:468-474.

16. Pantuck Aj, Zisman A, Belldegrun As. The changinh natural history of renal cell carcinoma. J. Urol. 2001; 166: 1611-1623.

17. Congregado Ruiz B, Medina López RA, Sánchez Gómez E, Morales López A, Pascual del Pobil JL. Diagnóstico incidental del carcinoma renal. ¿Implica un mejor pronóstico? Actas Urol Esp 2001;25:278-282.
18. Tsui KH, Shvarts O, Smith RB, Figlin RA, de Kernion JB, Belldegrun A. Prognostic indicators for renal cell carcinoma: a multivariate analysis of 643 patients using the revised 1997 TNM staging criteria. J Urol 2000;163:10901095.

19. Van Poppel H, Vandendriessche H, Boel K, Mertens V, Goethuys H, Haustermans K, Van Damme B, Baert L. Microscopic vascular invasion is the most relevant prognosticator after radical nephrectomy for clinically nonmetastatic renal cell carcinoma. J Urol 1997;158:4549.

20. Benitez R, Godelaine D, Lopez-Nevot MA, Brasseur F, Jimenez P, Marchand M, et al. Mutation of $\beta_{2}$-microglobulin gene result in lack of HLA class I molecules on melanoma cell of two patients inmunized with MAGE petptides. Tissue Antigens 1998;52:520-524.

Manuel Ortiz Gorraiz.

C/ Don Quijote 3, 3ํㅡ

18008 Granada

E-mail: mortig@fundacionhvn.org

(Trabajo recibido el 26 de septiembre de 2004) 